\title{
Examining the Difference between Migraine and Intracranial Arterial Stenosis Based on the Transcranial Doppler Findings
}

\author{
Hyeongseok Kim ${ }^{*}$, Seung-Jae Lee* (D), Aleum Lee ${ }^{\dagger}$ (D) \\ Departments of Neurology ${ }^{\star}$, Radiology ${ }^{\dagger}$, Soonchunhyang University Bucheon Hospital, Bucheon, Korea
}

Background: Data on the difference between migraine and intracranial arterial stenosis (ICAS) based on transcranial Doppler (TCD) findings are limited. This study aimed to investigate the differences in the features of TCD abnormality between migraine and ICAS.

Methods: Forty-one migraine patients with normal angiography and 35 ICAS patients were retrospectively recruited and assigned to two respective groups. The TCD variables from the arteries with Doppler abnormality were compared between the two groups using an independent $t$-test or chi-square test.

Results: For all analyzed intracranial arteries, turbulent flow was more frequent in the ICAS group. The prevalence of side-to-side difference $>30 \%$ was significantly higher in the middle cerebral artery (MCA) and vertebral artery (VA) of ICAS patients. Focal increase in mean flow velocity $(\mathrm{mFV})$ was more frequently observed in the MCA of ICAS patients. The $\mathrm{mFV}$ was markedly increased with a wide range of distribution in the MCA, VA, and basilar artery of ICAS patients. Meanwhile, the $\mathrm{mFV}$ in migraine patients was only modestly increased. The absence of turbulence flow had a sensitivity of $92.5 \%$ and a specificity of $88.9 \%$, while $\mathrm{mFV}<120 \mathrm{~cm} / \mathrm{s}$ had a sensitivity of $74.5 \%$ and a specificity of $100 \%$ to diagnose migraine.

Conclusion: Turbulent flow with a marked elevation, focal variation, and asymmetry of $\mathrm{mFV}$ favor the diagnosis of ICAS, while the relatively uniform and modest increase in $\mathrm{mFV}$ favor the diagnosis of migraine.

J Neurosonol Neuroimag 2019;11(2):132-13S

Key Words: Migraine; Doppler sonography; Transcranial; Intracranial arterial diseases
Received: October 7, 2019

Revised: October 31, 2019

Accepted: November 4, 2019

Address for correspondence: Seung-Jae Lee

Departments of Neurology, Soonchunhyang University Bucheon Hospital, 170 Jomaruro, Bucheon 14584, Korea Tel: $+82-32-621-6548$ Fax: $+82-32-621-6950$ E-mail:neurosj@catholic.ac.kr

\section{INTRODUCTION}

Transcranial Doppler (TCD) is frequently used as a substitute for brain angiography in a primary or outpatient setting. Practically, a number of TCD examinations are performed for explorative purpose in patients with complaint of headache. Of these, migraine patients frequently show abnormal Doppler findings before undergoing brain angiographies. ${ }^{1}$ In such cases, it is difficult to decide whether the abnormal Doppler findings indicate an undiagnosed intracranial arterial stenosis (ICAS) because many migraine patients exhibit an increase in flow velocity in the intracranial arteries without the presence of stenotic lesions. ${ }^{1,2}$ Apparently, most of the clinicians would obtain the angiographic data to determine the presence or absence of cerebrovascular abnormality in such patients.

To date, no study has compared the difference in abnormal Doppler findings between patients with migraine and those with ICAS. At present, it may be impossible to distinguish migraine from ICAS on the basis of Doppler abnormalities alone. Accordingly, in this study, we aimed to analyze the abnormal Doppler findings purely resulting from migraine pathophysi- 
ology by comparing the features of TCD abnormality between migraine and ICAS.

\section{PATIENTS AND METHODS}

\section{Study design and patients}

From the TCD registry, we initially obtained the names and registry numbers of 72 migraine patients who underwent brain computed tomographic angiography (CTA) and TCD on the same day from May 2015 to June 2018. From the selected patients, 25 with normal TCD, four with any discernible stenosis on CTA, and two with transtemporal approach failure were excluded to analyze the abnormal Doppler findings purely arising from migraine. Consequently, 41 migraine patients (including seven with aura) with abnormal TCD but normal CTA were included in the analysis.

Thirty-five patients with TCD abnormalities due to any discernible ICAS were recruited by reviewing the TCD registry and their medical records. We tried to match their ages with those of migraine patients. All ICAS patients underwent TCD within 1 week after undergoing brain CTA or magnetic resonance angiography (MRA) during the same period. The ICAS group consisted of 27 patients with large artery atherosclerosis, five with moyamoya disease, two with intracranial vertebrobasilar dissection, and one with cerebral vasculitis. This study was approved by the Institutional Review Board of Soonchunhyang University Bucheon Hospital (SCHB 2018-09-021), and the requirement for informed consent was waived due to the retrospective nature of our study.

\section{Clinical assessment}

The following clinical data were obtained: age, gender, hypertension, diabetes mellitus, hyperlipidemia, current smoking, coronary artery disease, blood pressure at the TCD study, and complete blood count (white blood cell count, hemoglobin/hematocrit, and platelet count) within 1 week of the TCD study. Migraine was diagnosed according to the criteria of the International Classification of Headache Disorders 2nd edition. ${ }^{3}$

\section{Imaging protocols}

Brain CTA was performed with a 128-detector high-definition CT scanner (Discovery CT750 HD; GE Healthcare, Milwaukee, Wisconsin, USA) using standardized protocol (section thickness, $0.625 \mathrm{~mm}$; tube voltage, $100 \mathrm{kV}$; and tube current, $300 \mathrm{~mA}$ ). Images were scanned after a single bolus injection of $100 \mathrm{~mL}$ of nonionic contrast agent via an 18-gauge cannula inserted into an antecubital vein. Images were obtained in series from the aortic arch to the vertex. Brain MRA was performed on a 3.0 Tesla MRI scanner (MAGNETOM Skyra; Siemens, Erlangen, Germany), and RF coil for head and neck examination (head/neck 64 channel coil; Siemens) was utilized. To obtain MRA images, a three-dimensional time-of-flight pulse sequence method was used. The brain CTA and MRA were interpreted by two experienced neuroradiologists including A.L.

\section{TCD protocol and variables}

TCD examination was performed in a quiet room according to previously recommended practice standards. ${ }^{4,5}$ Power M-mode TCD (ST3, Spencer Technologies, Seattle, WA, USA) was used for all examinations. Mean flow velocity ( $\mathrm{mFV}$ ) and pulsatile index (PI) of the siphon internal carotid artery (sICA) were recorded at a depth of 58-65 mm, middle cerebral artery (MCA) at 40-65 mm, anterior cerebral artery (ACA) at $60-75 \mathrm{~mm}$, posterior cerebral artery (PCA) at 55-75 $\mathrm{mm}$, vertebral artery (VA) at $40-75 \mathrm{~mm}$, and basilar artery (BA) at 8o$105 \mathrm{~mm}$. Two different experienced sonographers were blinded to the clinical data while performing the TCD studies. The results were interpreted through consensus among two investigators (S.J.L. and H.K.) according to previously published interpretation criteria., ${ }^{5,6}$ For the bilateral arteries, side-to-side differences (\%) in $\mathrm{mFV}$ were calculated by dividing the difference between the mFVs of bilateral arteries by the mFV of the lower value. Turbulent flow was defined as any definite increase in low-frequency components within the Doppler spectrum including bidirectional low-frequency noise and arterial wall covibration. For MCA, VA, and $\mathrm{BA}$, focal increase in $\mathrm{mFV}$ was determined as more than $30 \mathrm{~cm} / \mathrm{s}$ or $30 \%$ of the difference between arterial segments within the same artery (Fig. 1). 


\section{Statistical analysis}

Using independent $t$-test or chi-square test, the demographic variables, vascular risk factors, blood pressure, complete blood counts, and the TCD variables from the arteries with Doppler abnormality were compared between the migraine group and ICAS group. In addition, the absence of turbulent flow and mFV of $<120 \mathrm{~cm} / \mathrm{s}$ in any examined vessel were used to calculate the sensitivity and specificity for diagnosing migraine in the cohort. Statistical analyses were performed with
SPSS software, version 21.0 (SPSS Inc., Chicago, IL, USA). p values $<0.05$ were considered significant.

\section{RESULTS}

A total of 76 patients (41 migraine patients and 35 ICAS patients) were included in the analysis. The migraine group consisted of an overwhelmingly higher proportion of women (92.7\%), while the ICAS group had a higher proportion of men (62.9\%). Expectedly, the
Right MCA

A

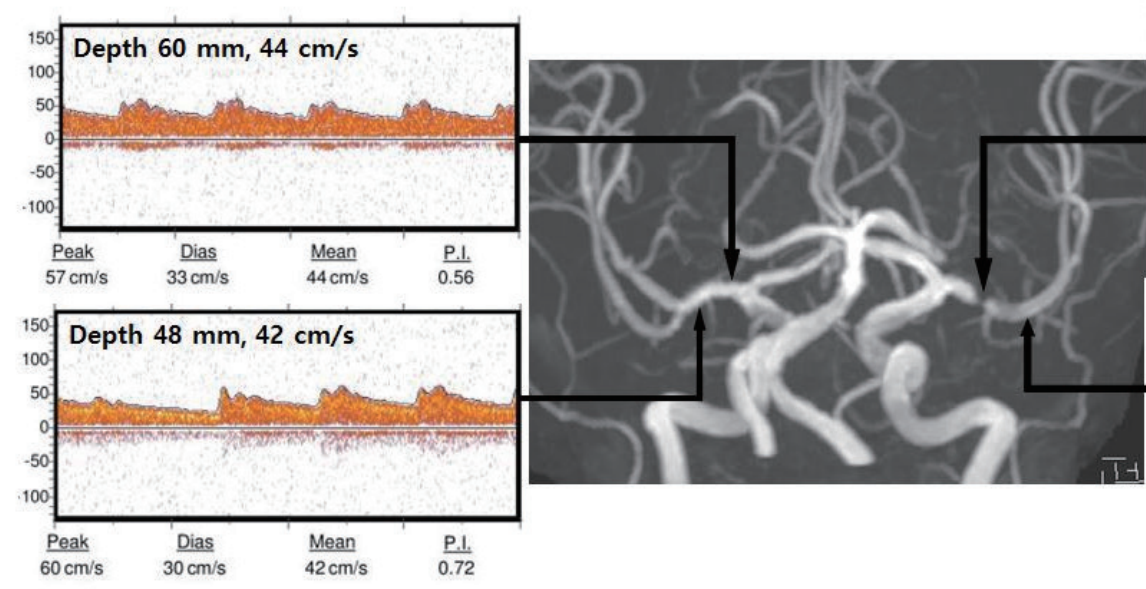

Left MCA

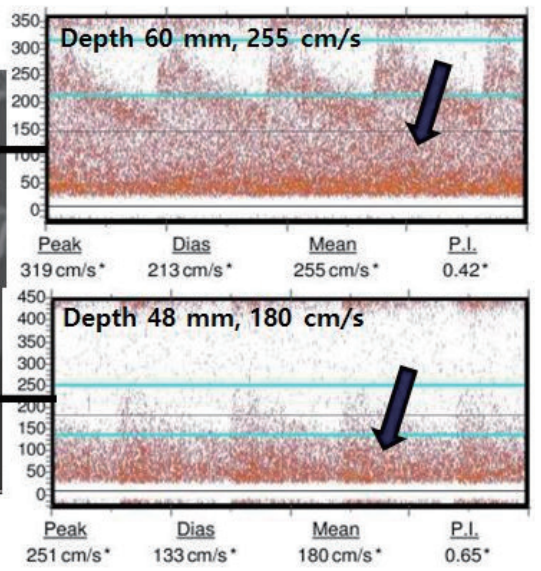

B
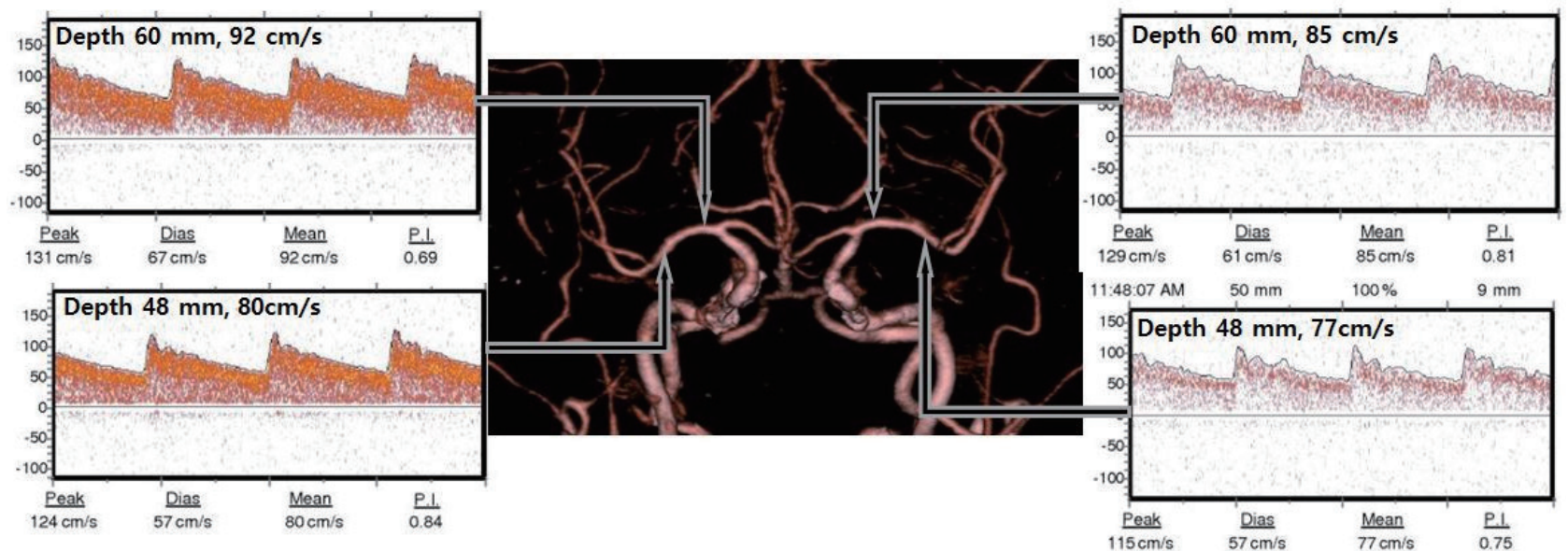

FIG. 1. Typical Doppler and angiography examples of intracranial arterial stenosis (A) and migraine (B). (A) Magnetic resonance angiography (upper center panel) shows focal severe stenosis at the left $\mathrm{Ml}$. Side-to-side differences (\%) in mean flow velocity (mFV) were calculated by dividing the difference between the mFVs of bilateral arteries by the $\mathrm{mFV}$ of the lower value. The value in this case was 479\% ([255-44]×100/44). Note the pattern of turbulent flow (black arrow, left upper panel), which was defined as any definite increase in low-frequency components within Doppler spectrum. Focal increase in mean flow velocity proved significant at the stenotic lesion of the left $\mathrm{M}_{1}$ since the difference of $\mathrm{mFV}$ between $\mathrm{M} \mathrm{I}$ segments of the same side was higher than $30 \mathrm{~cm} / \mathrm{s}([255-180]=75 \mathrm{~cm} / \mathrm{s})$ or $30 \%([255-180] \times 100 / 180=41.6 \%)$. (B) Computed tomographic angiography (lower center panel) shows normal vessels. Note the slight increase in $\mathrm{mFV}$ at the proximal segments of the bilateral middle cerebral arteries $(>80 \mathrm{~cm} / \mathrm{s})$. However, in contrast with (A), the side-to-side difference between bilateral homologous $\mathrm{M}$ segments is less than $30 \%$. No significant turbulent flow or focal increase in $\mathrm{mFV}$ was observed. 
ICAS group had a significantly higher prevalence of vascular risk factors including hypertension, diabetes, hyperlipidemia, and current smoking than the migraine group. Coronary artery disease tended to be frequent in the ICAS patients $(p=0.056)$. Additionally, age tended to be higher in the ICAS patients $(p=0.099)$, but the age difference between the two groups was not statistically significant. White blood cell count, hemoglobin, hematocrit, and systolic and diastolic blood pressures were significantly higher in the ICAS group (Table 1).

In the 41 migraine patients, the most frequent vessel with TCD abnormality was MCA (32 patients, 78.0\%), followed by VA (26 patients, 63.4\%), sICA (10 patients, 24.4\%), BA (eight patients, 19.5\%), ACA (six patients, 14.6\%), and PCA (o patient). Among the 35 ICAS patients, the most frequent vessel with Doppler abnormality was MCA (27 patients, 77.1\%), followed by sICA (15 patients, 42.9\%), ACA (15 patients, 42.9\%), VA (11 patients, 31.4\%), BA (seven patients, 20.0\%), and PCA (one patient, 2.9\%) (Fig. 2). Therefore, PCA was excluded from the analysis.

Table 2 shows a comparative analysis of Doppler findings between patients with migraine and those with ICAS. For all the analyzed intracranial arteries, turbulent flow was significantly more frequent in the ICAS group than in the migraine group. The prevalence of side-to-side difference $>30 \%$ was significantly higher in the MCA and VA of the ICAS patients than in those of migraine patients, but the prevalence of side to side difference $>30 \%$ in the sICA and ACA was statistically similar between the two groups.

Focally increased $\mathrm{mFV}$ was more frequently observed in the MCA of ICAS patients than in that of migraine patients, but there was no significant difference in the BA and VA between the two groups. Compared with that of migraine patients, the mFV of ICAS patients not only had a wide range of distribution, but was also significantly higher in the MCA, VA, and BA, and was non-significantly $(p<0.1)$ increased in the sICA and ACA.

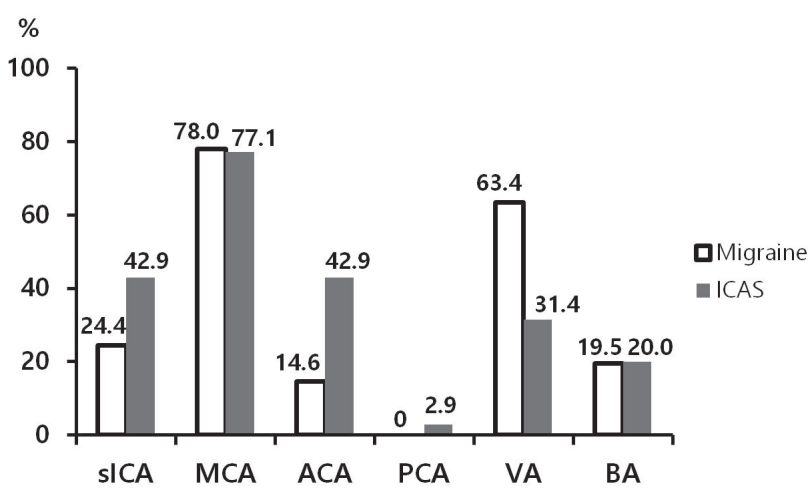

FIG. 2. Prevalence of arteries with Doppler abnormality in patients with migraine and intracranial arterial stenosis (ICAS). sICA; siphon internal carotid artery, MCA; middle cerebral artery, ACA; anterior cerebral artery, PCA; posterior cerebral artery, VA; vertebral artery, BA; basilar artery.

TABLE 1. Basic characteristics of patients with migraine vs. intracranial arterial stenosis

\begin{tabular}{lccc}
\hline & Migraine $(n=41)$ & Intracranial arterial stenosis $(n=35)$ & $p$-value \\
\hline Age (years) & $41.3 \pm 9.8$ & $45.1 \pm 9.7$ & 0.099 \\
Female & $38(92.7)$ & $13(37.1)$ & $<0.001$ \\
Hypertension & $4(9.8)$ & $27(77.1)$ & $<0.001$ \\
Diabetes & $0(0)$ & $14(40.0)$ & $<0.001$ \\
Hyperlipidemia & $0(0)$ & $24(68.6)$ & $<0.001$ \\
Current smoking & $3(7.3)$ & $12(34.3)$ & 0.003 \\
Coronary artery disease & $0(0)$ & $3(8.6)$ & 0.056 \\
Systolic blood pressure $(\mathrm{mmHg})$ & $120.6 \pm 15.0$ & $150.1 \pm 22.2$ & $<0.001$ \\
Diastolic blood pressure $(\mathrm{mmHg})$ & $76.9 \pm 13.0$ & $91.0 \pm 17.5$ & $<0.001$ \\
Complete blood count & & & 0.015 \\
White blood cell $\left(\times 10^{9} / \mathrm{L}\right)$ & $7.0 \pm 2.4$ & $14.6 \pm 2.7$ & 0.020 \\
Hemoglobin $(\mathrm{g} / \mathrm{dL})$ & $13.1 \pm 1.4$ & $41.0 \pm 3.6$ & 0.024 \\
Hematocrit $(\%)$ & $38.7 \pm 4.4$ & $258.1 \pm 102.5$ & 0.659 \\
Platelet count $\left(\times 10^{9} / \mathrm{L}\right)$ & $267.9 \pm 54.6$ & & \\
\hline
\end{tabular}

Variables were analyzed using independent $t$-test (mean \pm standard deviation) or chi-square test (n [\%]). 
On the contrary, the mFV in migraine patients was only moderately increased: the maximum value was below $95 \mathrm{~cm} / \mathrm{s}$ in the sICA and ACA, $120 \mathrm{~cm} / \mathrm{s}$ in the MCA, and $85 \mathrm{~cm} / \mathrm{s}$ in the BA and VA. PI was nonsignificantly higher in the VA of ICAS patients, but was significantly increased in the ACA of ICAS patients compared with their counterpart.

The absence of turbulent flow and $\mathrm{mFV}<120 \mathrm{~cm} / \mathrm{s}$ in any examined vessel were used to calculate the sensitivity and specificity for the diagnosis of migraine in our cohort. The absence of turbulence flow had a sensitivity of $92.5 \%$ and a specificity of $88.9 \%$, while $\mathrm{mFV}<120 \mathrm{~cm} / \mathrm{s}$ had a sensitivity of $74.5 \%$ and a specificity of $100 \%$ to diagnose migraine.

TABLE 2. Comparison of Doppler findings between patients with migraine vs. ICAS

\begin{tabular}{|c|c|c|c|}
\hline Vessels with Doppler abnormality & Migraine & ICAS & $p$-value \\
\hline Number of sICA & 13 & 21 & \\
\hline $\mathrm{mFV}(\mathrm{cm} / \mathrm{s})^{*}$ & $78.5 \pm 6.3(72-92)$ & $87.6 \pm 22.7(53-146)$ & 0.097 \\
\hline Pulsatile index & $0.68 \pm 0.15(0.42-0.98)$ & $0.67 \pm 0.16(0.40-0.93)$ & 0.825 \\
\hline Side to side difference $>30 \%$ & $3(23.1)$ & $6(28.6)$ & 1.000 \\
\hline Turbulent flow & $2(15.4)$ & $17(81.0)$ & $<0.001$ \\
\hline Number of MCA & 54 & 44 & \\
\hline $\mathrm{mFV}(\mathrm{cm} / \mathrm{s})^{*}$ & $90.4 \pm 7.5(81-116)$ & $140.8 \pm 62.7(67-299)$ & $<0.001$ \\
\hline Pulsatile index & $0.65 \pm 0.11(0.44-0.93)$ & $0.68 \pm 0.18(0.20-1.08)$ & 0.337 \\
\hline Side to side difference $>30 \%$ & $\mathrm{o}(0)$ & $32(72.7)$ & $<0.001$ \\
\hline Turbulent flow & $2(3.7)$ & $36(81.8)$ & $<0.001$ \\
\hline Focal increase in $\mathrm{mFV}^{\dagger}$ & $6(11.1)$ & $25(56.8)$ & $<0.001$ \\
\hline Number of ACA & 7 & 21 & \\
\hline $\mathrm{mFV}(\mathrm{cm} / \mathrm{s})^{*}$ & $86.3 \pm 4.7(81-94)$ & $107.5 \pm 28.0(79-199)$ & 0.059 \\
\hline Pulsatile index & $0.58 \pm 0.10(0.43-0.74)$ & $0.75 \pm 0.17(0.54-1.25)$ & 0.017 \\
\hline Side to side difference $>30 \%$ & $3(42.9)$ & $8(38.1)$ & 1.000 \\
\hline Turbulent flow & $\mathrm{o}(0)$ & $15(71.4)$ & 0.001 \\
\hline Number of VA & 39 & 18 & \\
\hline $\mathrm{mFV}(\mathrm{cm} / \mathrm{s})^{*}$ & $58.5 \pm 7.2(50-83)$ & $108.6 \pm 58.3(51-251)$ & 0.002 \\
\hline Pulsatile index & $0.66 \pm 0.12(0.46-0.91)$ & $0.72 \pm 0.10(0.61-0.95)$ & 0.075 \\
\hline Side to side difference $>30 \%$ & $3(7 \cdot 7)$ & $9(50.0)$ & 0.001 \\
\hline Turbulent flow & $\mathrm{o}(0)$ & $15(83 \cdot 3)$ & $<0.001$ \\
\hline Focal increase in $\mathrm{mFV}^{\dagger}$ & $21(53.8)$ & $12(66.7)$ & 0.362 \\
\hline Number of BA & 8 & 7 & \\
\hline $\mathrm{mFV}(\mathrm{cm} / \mathrm{s})^{*}$ & $71.9 \pm 7.4(63-81)$ & $103 \cdot 3 \pm 29 \cdot 3(64-142)$ & 0.030 \\
\hline Pulsatile index & $0.69 \pm 0.9(0.56-0.81)$ & $0.76 \pm 0.19(0.52-1.00)$ & 0.378 \\
\hline Turbulent flow & $\mathrm{o}(\mathrm{o})$ & $4(57.1)$ & 0.026 \\
\hline Focal increase in $\mathrm{mFV}^{\dagger}$ & $2(25 \cdot 0)$ & $4(57.1)$ & 0.315 \\
\hline
\end{tabular}

Variables were analyzed using independent $t$-test (mean \pm standard deviation [range]) or chi-square test $(\mathrm{n}[\%])$.

ICAS; intracranial arterial stenosis, sICA; siphon internal carotid artery, mFV; mean flow velocity, MCA; middle cerebral artery, ACA; anterior cerebral artery, VA; vertebral artery, BA; basilar artery

${ }^{*}$ Maximum mean flow velocity at each vessel was used.

${ }^{\dagger}$ Focal increase in mean flow velocity was defined as $30 \mathrm{~cm} / \mathrm{s}$ or $30 \%$ of the difference between arterial segments within the same artery. 


\section{DISCUSSION}

For all the analyzed arteries, turbulent flow was the persistent feature of ICAS distinct from migraine. It can be explained by the fact that TCD directly insonates stenosed large arteries in the ICAS patients. The stenosis geometry is prone to cause disturbed flow rather than smooth laminar flow.7 Furthermore, the localized and lateralized nature of stenotic lesions is more likely to cause a higher prevalence of side-to-side difference and focal increase in mFV in ICAS. The features, in particular, were well presented in the MCAs of ICAS patients, which are not only less variant, but also have a straight course allowing a favorable angle of insonation.

In contrast, increased flow velocity in migraine patients is assumed to be attributable to arteriolar vasodilatation rather than reduced lumen diameter in the insonated large arteries. This stands to reason in our study because we recruited migraine patients with normal CTA, which was performed on the same day with TCD. As previously reported, increased neuronal excitability and neurovascular coupling in migraine $e^{8,9}$ could be linked with reduced arteriolar resistance and increased regional blood flow, ${ }^{10}$ which can result in the increased flow velocity in the insonated large arteries. ${ }^{11}$ From the pathophysiological aspect, even increase in flow velocity along the large arteries is anticipated, and thus turbulent flow and focal increase in $\mathrm{mFV}$ might well be less prevalent in migraine patients.

The mFV was significantly or non-significantly higher in ICAS patients. According to a simple model of the continuity principle, flow velocity increases by an amount inversely proportional to the reduction of arterial lumen area if other factors (e.g., collateral channel, blood viscosity, blood pressure) are excluded.? Therefore, the flow velocity in ICAS can markedly increase with a wide distribution of values as the stenosis progresses. By contrast, the $\mathrm{mFV}$ in migraine was only modestly increased in line with previous studies (Fig. 1). ${ }^{2}$

Focally increased $\mathrm{mFV}$ was more frequently found in the MCA of ICAS patients than in that of migraine patients. However, there was no significant difference in the prevalence in the VA and BA between the two groups. Particularly, 53.8\% of VA in migraine patients had a focal increase in $\mathrm{mFV}$. It may be because of the frequent tortuous courses with morphological varia- tions in posterior circulation arteries in addition to the incapability of angle correction in the TCD machine used in the study. ${ }^{12,13}$ For this reason, VA is one of the arteries with the lowest sensitivity for TCD diagnosis of its stenosis. ${ }^{14}$

In summary, turbulent flow with a marked elevation, focal variation, and asymmetry of $\mathrm{mFV}$ favor the diagnosis of ICAS, while a relatively uniform and mild to moderate increase in $\mathrm{mFV}$ favor the diagnosis of migraine if other influencing factors are excluded. The results seem to reflect the different locations where the luminal diameter of vessels changes in each disease entity.

To the best of our knowledge, this is the first study to compare the abnormal TCD findings of migraine patients with those of ICAS patients. This approach can provide a useful insight to interpret the Doppler findings of patients with migraine from the pathophysiological aspect and thus bring out a further clinical use of TCD in real-world headache practice. However, this study has some limitations. It was based on a small number of data obtained from a single center. Therefore, the statistical power is too low for multivariable analysis. Besides, age could not be completely matched in the process of patient selection. That is to say, the patients in the ICAS group tended to be older $(p=0.99)$. This is because the ICAS patients in the Soonchunhyang University Bucheon Hospital are mostly older individuals. The incompleteness in age matching might have led to the differences in the results for some measurements (e.g., increased PI) in the present study. Additionally, there were differences in blood pressure and blood constituents (hemoglobin and hematocrit) between the two groups. It could have affected flow velocity and PI in our data. ${ }^{7,15}$ In addition, we could not determine if it was ictal or inter-ictal phase when TCD was performed in the migraine patients because of the retrospective nature of our study. As previous studies have demonstrated an increased flow velocity during the headache-free period, ${ }^{1,2,11,16}$ the migraine status of most of our patients are believed to have corresponded to the inter-ictal phase of migraine.

\section{Acknowledgements}

The authors are thankful to Dr. Tae-Kyeong Lee and Eek-Sung Lee for their contribution to the collection of 
data.

This work was supported by the Soonchunhyang University Research Fund.

\section{Conflicts of Interest}

No potential conflicts of interest relevant to this article was reported.

\section{REFERENCES}

1. Hong SN, Kim BK, Koo JS, Lee YS, Bae HJ. Clinical correlation of abnormal transcranial Doppler in migraineurs. J Korean Neurol Assoc. 2000;18:711-715.

2. Arjona A, de Torres LA, Serrano-Castro PJ, Guardado-Santervas PL, Olivares J, Rubí-Callejon J. A transcranial Doppler study in interictal migraine and tension-type headache. J Clin Ultrasound. 2007;35:372-375.

3. Headache Classification Subcommittee of the International Headache Society. The international classification of headache disorders: 2nd edition. Cephalalgia. 2004;24 Suppl 1:9160.

4. Alexandrov AV, Sloan MA, Wong LK, Douville C, Razumovsky AY, Koroshetz WJ, et al. Practice standards for transcranial Doppler ultrasound: part I--test performance. J Neuroimaging. 2007;17:11-18.

5. Lee JY, Yu S, Lee SI, Jung KH, Seo WK, Park JM, et al. Transcranial Doppler ultrasound: practice standards part I. Test performance and interpretation. J Neurosonol. 2016;8:1-13.

6. Alexandrov AV, Neumyer MM. Diagnostic criteria for cerebrovascular ultrasound. In: Alexandrov AV. Cerebrovascular ultrasound in stroke prevention and treatment. 2nd ed. New York: Wiley. 2011;87-143.

7. Alexandrov AV. Practical models of cerebral hemodynamics and waveform recognition. In: Alexandrov AV. Cerebrovascular ultrasound in stroke prevention and treatment. 2nd ed. New York: Wiley. 2011;68-84.
8. Brighina F, Piazza A, Daniele O, Fierro B. Modulation of visual cortical excitability in migraine with aura: effects of $1 \mathrm{~Hz}$ repetitive transcranial magnetic stimulation. Exp Brain Res. 2002;145:177-181.

9. Zaletel M, Strucl M, Bajrović FF, Pogacnik T. Coupling between visual evoked cerebral blood flow velocity responses and visual evoked potentials in migraneurs. Cephalalgia. 2005;25:567-574.

10. Loehrer E, Vernooij MW, van der Lugt A, Hofman A, Ikram MA. Migraine and cerebral blood flow in the general population. Cephalalgia. 2015;35:190-198.

11. Lee MJ, Chu MK, Choi H, Choi HA, Lee C, Chung CS. Longitudinal changes in cerebral blood flow velocities in different clinical courses of migraine. Cephalalgia. 2017;37:927937.

12. Park JA, Jung KH, Kim JM, Lee WJ, Ko SB, Lee SH, et al. Comparative study of transcranial color-coded Doppler and transcranial Doppler sonography in middle cerebral artery. J Neurosonol Neuroimag. 2018;10:25-33.

13. Krejza J, Mariak Z, Babikian VL. Importance of angle correction in the measurement of blood flow velocity with transcranial Doppler sonography. AJNR Am J Neuroradiol. 2001;22:1743-1747.

14. Guan J, Zhou Q, Ouyang H, Zhang S, Lu Z. The diagnostic accuracy of TCD for intracranial arterial stenosis/occlusion in patients with acute ischemic stroke: the importance of time interval between detection of TCD and CTA. Neurol Res. 2013;35:930-936.

15. Alexandrov AW. Integrated assessment of systemic and intracranial hemodynamics. In: Alexandrov AV. Cerebrovascular ultrasound in stroke prevention and treatment. 2nd ed. New York: Wiley. 2011;47-67.

16. Shayestagul NA, Christensen CE, Amin FM, Ashina S, Ashina M. Measurement of blood flow velocity in the middle cerebral artery during spontaneous migraine attacks: a systematic review. Headache. 2017;57:852-861. 